\title{
On the interaction of a racing car front wing and exposed wheel
}

\section{S. Diasinos}

sammy.diasinos@mq.edu.au

Macquarie University

North Ryde

New South Wales

Australia

G. Doig

g.doig@unsw.edu.au

School of Mechanical and Manufacturing Engineering

The University of New South Wales

Sydney, New South Wales

Australia

\section{T. J. Barber}

t.barber@unsw.edu.au

Associate Professor

School of Mechanical and Manufacturing Engineering

The University of New South Wales

Sydney, New South Wales

Australia

\section{ABSTRACT}

A numerical investigation of generic open-wheel racing car wing and wheel geometry has been conducted, using original sub-scale experimental data for validation. It was determined that there are three main interactions that may occur, identifiable by the path that the main and secondary wing vortices take around the wheel. Interaction ' $A$ ' occurs when the main and secondary wing vortices both travel outboard of the wheel; interaction ' $\mathrm{B}$ ' is obtained when only the main wing vortex passes inboard of the wheel; while interaction ' $C$ ' sees both wing vortices travel inboard of the wheel. The different interactions are achieved when geometric changes to the wing affect the pressure distribution about the endplate, either by altering the magnitude of suction generated by the wing or by changing the locations of peak suction and vortices relative to the wheel's stagnation regions. As a result, the influence that the wing and wheel have on each other - in comparison to the same bodies in isolation - varies, resulting in significant consequences for downforce and drag. 


\section{NOMENCLATURE}

$\alpha$ angle-of-attack

$c$ chord

$C_{D}$ coefficient of drag

$C_{L}$ coefficient of negative lift (downforce)

$C_{P T}$ coefficient of total pressure

$\varepsilon \quad$ turbulent dissipation

$h$ minimum height above ground plane

I turbulent intensity

$k$ turbulent kinetic energy

$T$ Total width of wing and wheel arrangement

$v_{\infty}$ freestream velocity

$W$ wheel width

$\omega$ specific dissipation rate

\subsection{INTRODUCTION}

The performance of current open wheeler race cars depends heavily on the effectiveness of the aerodynamic package. Numerous components including wings, floor, diffuser, barge boards and splitter plate are all used to generate downforce. The ultimate goal is to optimise the shape of these appendages within the scope of a given set of regulations in order to extract the maximum possible downforce while also improving the vehicle's aerodynamic efficiency ${ }^{(1)}$.

On modern open wheel racing cars, approximately $30 \%$ of the total downforce originates from the front wing ${ }^{(2)}$, and its wake is likely to influence the performance of aerodynamic components located downstream ${ }^{(3)}$. Due to the requirements of regulations, the wheels are exposed and therefore can contribute up to $40 \%$ of the vehicle's total drag ${ }^{(4)}$. The aerodynamic performance of front wings in isolation has been studied extensively in recent years ${ }^{(5-10)}$, and several other reports on isolated.wheels have also increased knowledge about wheel performance when free of wing interference ${ }^{(11-15)}$. Intuitively, the close proximity of the front wheels to the front wing would be expected to have a strong influence on the overall system performance, yet the physics associated with the interaction of these two components remains poorly understood ${ }^{(1)}$. Indeed, in 2006 an otherwise comprehensive review of aerodynamic studies relevant to open wheel racing cars was presented by Zhang et $a l^{(16)}$; no mention was made of any investigations concerning wing and wheel interactions and it was suggested that greater research is required on this specific topic.

Agathangelou and Gascoyne.suggested that experiments alone would not be enough to understand this complicated flowfield ${ }^{(4)}$; Kellar et al undertook a numerical and experimental study a combined wing and wheel interaction ${ }^{(17)}$. Unfortunately, no useful detailed information of the wing and wheel geometry was provided, but from figures approximately a quarter of the wheel was overlapped by the wing. A rigid board represented the ground plane - an approximation which may have significantly influenced the results due to unrealistic boundary-layer growth ${ }^{(18)}$. This raised board featured a cut out which the wheel dropped into, allowing a mechanism below the board to drive the wheel rotation. Such a ground representation has been demonstrated to adversely influence the results of investigations concerning both isolated downforce producing wings ${ }^{(19)}$, and isolated wheels ${ }^{(20)}$, in ground effect. Nevertheless, the results indicated that the removal of the front wing caused the wheel drag to increase by between $10 \%$ and $20 \%$ depending on endplate geometry. Smoke visualisation indicated that the wing tip vortex shed from the bottom edge of the endplate 
travelled across the wheel tread and along the inner face. The reduced drag was hypothesised to be caused by the increase in vorticity created by the front wing, but contradictory to this assumption, the endplate which produced the weakest vortex produced the greatest drag reduction.

The accompanying computational model used by Kellar $e t$ al used a $k-\varepsilon$ turbulence closure which also allowed for compressibility ${ }^{(17)}$. They reported a requirement that the computational model be solved with a freestream value of Mach $0 \cdot 3$ in order to achieve convergence, which is greater than the highest speeds typically obtained by an open wheel racing car and may have inadvertently introduced complex Mach and Reynolds number interference effects ${ }^{(21-24)}$. No comparisons were made to the experimental results as a result but, qualitative results indicated that the stagnation point at the most upstream location of the wheel can be influenced by the endplate geometry.

An experimental investigation of open-wheeler aerodynamics was conducted by Katz ${ }^{(25)}$, including the effect of increasing front wing downforce on total vehicle downforce of a $25 \%$ scale model. An elevated ground rather than a rolling road was used to represent the ground. Increasing the front wing downforce by increasing the angle-of-attack of the second element of the front wing consistently moved the centre of pressure of the vehicle forward. This change produced a non linear relationship with the total car's downforce, as initial changes in the flap angle-of-attack increased the total downforce before a peak was reached and subsequent increases in flap angle-of-attack resulted in reductions. It was believed that increasing the downforce of the front wing adversely affected the performance of the remaining aerodynamic components downstream.

An experimental study by Diasinos and Gatto using laser doppler anemometry (LDA) on a generic wing and wheel combination ${ }^{(26)}$, albeit at a Reynolds number significantly lower than a full-scale car (Reynolds number, based on wing chord, of $5 \cdot 11 \times 10^{4}$ ), indicated that the angle-of-attack and wing span both had significant influence on the downstream wake behaviour. The primary, or main vortex produced on the lower surface at the endplate travelled inside of the wheel when the wing span was short, producing an asymmetric wheel wake with a broad clockwise rotational tendency. At larger spans, the opposite was found, with the primary vortex travelling outside of the wheel. Increasing angle-of-attack caused the strength and size of the vortices to increase. These experiments were used for validation of the present numerical model and are discussed further in subsequent sections.

While those experiments were referred to as 'sub-scale', the qualitative findings were confirmed by Van den Berg and Zhang ${ }^{(27)}$, who conducted a detailed experimental study on a wing/wheel combination based on a mid-2000s F1 design with grooved tyres. This remains the only significant full-scale study of its kind in public literature to the best of the authors' knowledge. A moving ground was present in the wind tunnel, and tests were run at $30 \mathrm{~ms}^{-1}$, matching previous front wing work conducted in the same tunnel ${ }^{(6-10)}$. A generic double element wing was used with vertical endplates, and the wheels were rigid carbon fibre with a small amount of camber. The sides of the wheel were covered to be flat, potentially simplifying the model and the flowfield ${ }^{(28)}$. Force and pressure measurements, as well as PIV and surface oil-flow visualisation were used to generate comprehensive results. However the results focus exclusively on the effect of changing ride height, keeping the wing and wheel overlap constant as well as the wing angle-of-attack and other parameters. It was discovered that high ground clearances resulted in reduced lift for the wing, and higher drag for both the wing and wheel. At low clearances the wing efficiency improved and the wheel drag was reduced. The trajectory of the top edge vortex was determined to be critical to performance.

Clearly, several complex interactions can occur around a front wing and wheel arrangement, but there persists an incomplete characterisation and understanding of the flowfields. The research described here is aimed at providing an overview categorisation of all possible main vortex path scenarios, and there follows a discussion of consequences for performance and wake behaviour. 


\subsection{NUMERICAL MODEL}

\subsection{Geometry and setup}

The experiments by Diasinos and Gatto were detailed enough to allow useful validation of the present numerical approach in terms of choice of turbulence model to describe the complexities of the wake, despite the scale being an order of magnitude below that of an actual racing car $^{(25)}$. Subsequent to this, a full-scale wing and wheel model was constructed to investigate the flow features at a more appropriate Reynolds number. All CFD results presented here were generated using Reynolds-Averaged Navier Stokes (RANS) modelling in ANSYS Fluent, a commercial code used by several teams in Formula 1. A pressure-based segregated solver was implemented using the SIMPLEC algorithm, with second order upwind discretisation applied. All gradients were evaluated at the cell centres and a converged solution was deemed to be obtained only when there was negligible variation in the force coefficients with extensive continued iteration. Only steady-state simulations were considered; this remains the preferred approach in industry for reasons of computational expense and real-time limits, despite the obvious propensity for racing car component flowfields (particularly around the wheels) to be highly unsteady ${ }^{(14)}$.

In brief, the wind-tunnel scale wing was an inverted, single-element NACA4412 section (chord, $c=75 \mathrm{~mm}$ ) with no taper or sweep; a rectangular endplate featuring a rounded leading and trailing edge was fitted to the tip. The endplate was fixed at $0.04 \mathrm{c}$ below the lowest point of the wing with the ride height of the wing measured as the minimum distance from the wing assembly to the ground. The minimum distance between the endplate and the foremost point of the wheel was fixed at $0.087 c$. A wheel diameter and width of $88 \mathrm{~mm}(1 \cdot 14 c)$ and $47 \cdot 3 \mathrm{~mm}(0 \cdot 61 c)$ were used, respectively. Wheel shoulders of radius equal to $0 \cdot 067 c$ were applied symmetrically - key features are detailed in Fig. 1. A bespoke sting was designed for minimum flow interference, and all tests were conducted at a freestream velocity of $10 \mathrm{~ms}^{-1}$. Full details of the experiments and the windtunnel design and test section, etc. may be found in literature ${ }^{(26,28)}$ - results are presented as-is for the validation study described here, and the model reproduced the test section geometry, inlet conditions and turbulence levels as faithfully as possible. A reference schematic of the tunnel setup is shown in Fig. 2, with evaluation plane locations noted for reference for later figures.

Mesh convergence studies were undertaken with the $k$ - $\varepsilon$ realisable model. Additionally, the wing height, span and angle-of-attack values during this verification step were maintained at $0 \cdot 13 c, 1 \cdot 42 c$ and $8^{\circ}$ respectively, while the wheel track was $1 \cdot 6 c$. Choice of turbulence model was evaluated subsequent to this step. The wind-tunnel dimensions, including the moving ground and wheel sting

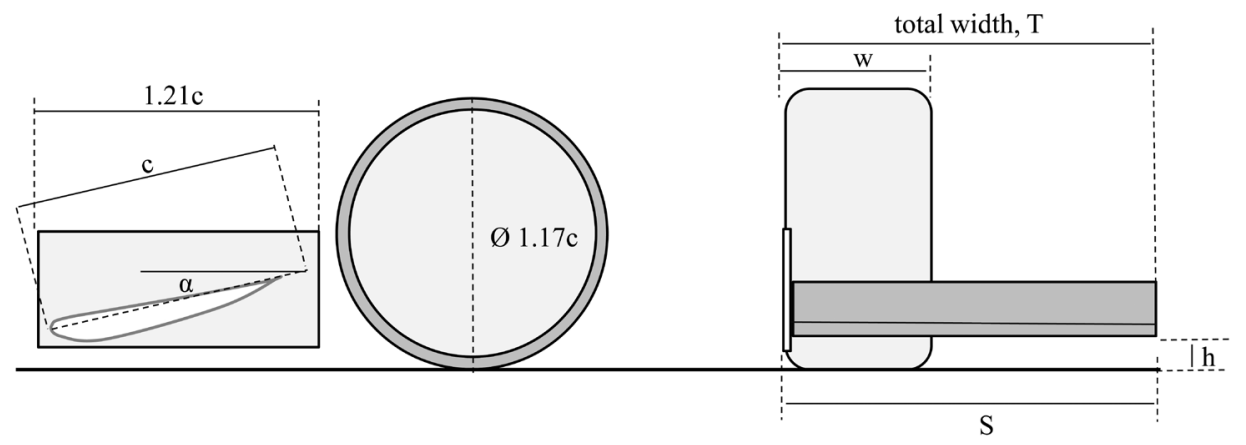

Figure 1. Schematic of wing and wheel geometry with relevant dimensions. 


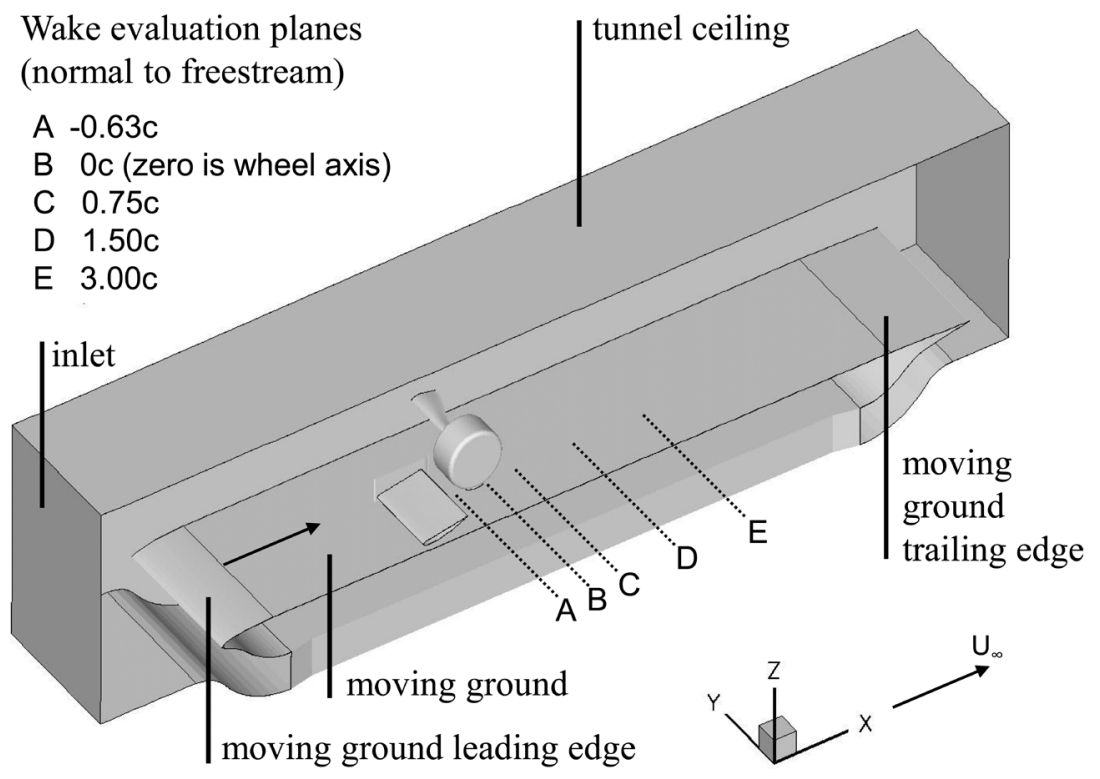

Figure 2. Sub-scale model in wind tunnel with wake evaluation plane locations.

features, were modelled as precisely as possible to improve correlation (Fig. 1). Independent wing and wheel validation was also undertaken against the experiments of Zerihan ${ }^{(6-10)}$ and Fackrell ${ }^{(11)}$ respectively, but is not detailed here for the sake of brevity - these cases may be found in other literature $^{(5,6,27)}$. For the scaled model of the wind-tunnel setup, the inlet was positioned at the start of the test section, nine chord lengths upstream of the wing. The outlet was positioned in the same relative location as that of the full scale model (15.6c downstream of the wheel rotation axis) which required the rear of the test section to be extruded rearward. The only additional modifications made to the boundaries of the scaled computational model relative to the full scale computational model were; the removal of the symmetry plane which was replaced by the left wind-tunnel wall, and the inclusion of the wheel sting as this was expected to have an effect on the wake structures measured by the LDA.

Subsequent to validation and verification steps, a full-scale wing and wheel (with chord and diameter of $562.5 \mathrm{~mm}$ and $660 \mathrm{~mm}$ respectively) were investigated with an inlet velocity of $33.3 \mathrm{~ms}^{-1}$. This equates to a Reynolds number of $1.28 \times 10^{6}$ based on the wing chord and $1.50 \times 10^{6}$ based on the wheel diameter. The full-scale computational model was developed to ensure that the wing and the wheel were modelled without any constraints or influence from wind-tunnel walls or the sub-scale Reynolds number. Both the upper and side boundaries were modelled as walls with zero shear while the ground was modelled as a smooth no-slip moving wall with a velocity equal to that of the freestream velocity. An angular velocity of $100 \cdot 9 \mathrm{rads}^{-1}$ was applied to the wheel boundary about its own axis so that the wheel's tangential velocity at the circumference was equal to that of the freestream velocity and also produced a zero slip condition with the ground at the contact patch. In order to reduce the size of the computational model, a symmetry plane was used allowing half the wing and only a single wheel to be modelled. The inlets in all cases featured constant turbulence intensity, I, of $0 \cdot 15 \%$, identical to LDA measurements of tunnel conditions. 


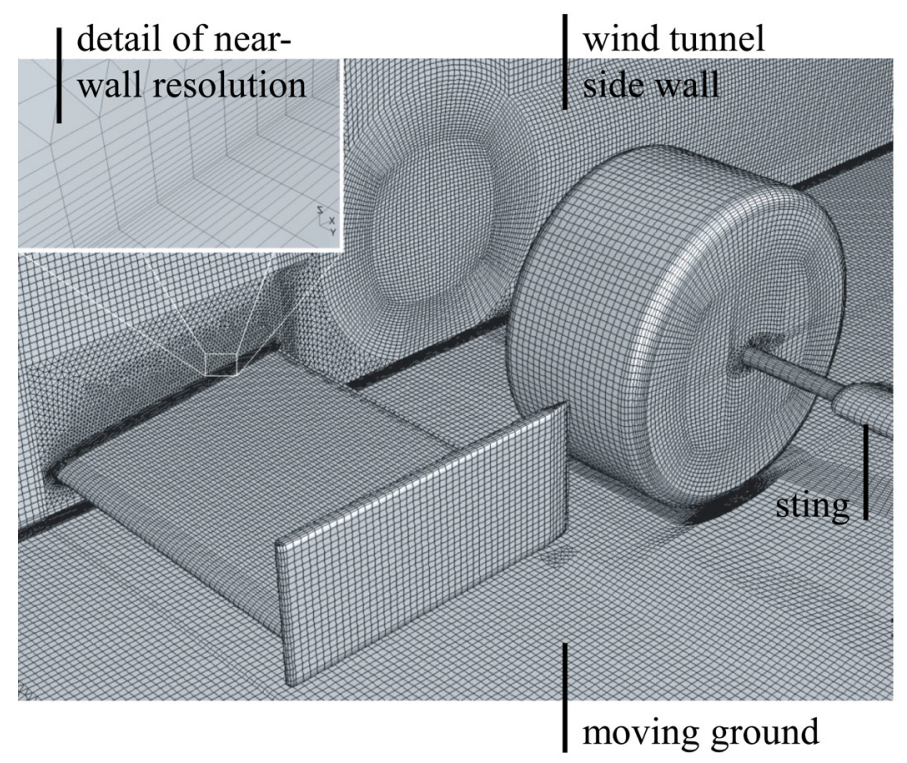

Figure 3. Example surface mesh for the wind-tunnel sub-scale wing and wheel model.

\subsection{Mesh description and verification}

The grid - the general approach of which is shown in part in Fig. 3 - was constructed in multi-block structured fashion, designed to allow flexiblity and variation in key regions (near-wall, wake, etc) for refinement studies. Some semi-structured (tetrahedral face mesh extruded with regularity) mesh around the wing and parts of the wheel was used, accounting for a very small percentage of the total number of cells.

To obtain a grid-independent result, models were created with a total number of cells ranging from $4.5 \times 10^{6}$ to $15.5 \times 10^{6}$. The variation in the lift and drag of the wing and wheel due to the mesh size was calculated by comparing each result obtained to that from the model with the smallest characteristic length and therefore the largest number of control volumes (Fig. 4) as suggested by the AIAA ${ }^{(28)}$. The critical near-wall region was defined by a $y^{+}$of 1.5 or less, enabling high-resolution near-wall modelling. A mesh with approximately $8 \times 10^{6}$ cells did not affect the lift and drag of either the wing and the wheel by more than $1.5 \%$ in comparison to a computational model with twice the number of cells; this was considered to be a suitable compromise between accuracy and the computational resources required to obtain a solution.

Given that the study involves examination of the flow features in the wake produced by the wing, wheel, and combinations of both, a more detailed comparison between results on the different meshes was also made to determine the differences in wake velocities which were observed on multiple planes (at the same non-dimensional locations for the full-scale cases as at B through E from Fig. 2). The variances are indicated in Fig. 5, and highlight the relatively small discrepancies present in areas of strong velocity gradients (particularly in the vicinity of the major vortex structures). The most notable differences occur on the outside edge of the wheel, in the vicinity of the area through which flow from the front wing endplate travels around the outside of the wheel in the right circumstances. Given the agreement demonstrated for the majority of the flowfield and for other parameters, this was deemed to be acceptable. 


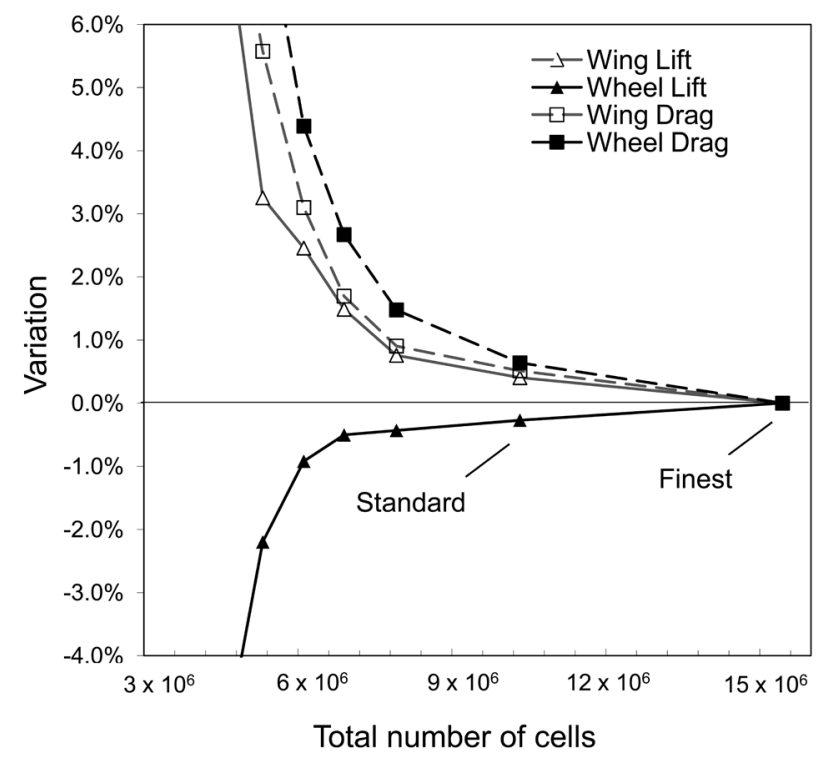

Figure 4. Lift and drag on the wing and wheel with increasing mesh cell density.

\subsection{Selection of turbulence model}

Two two-equation turbulence models with accepted capability for adequately resolving separated flows were chosen for comparison; the realisable $k$ - $\varepsilon^{(30)}$ and $k-\omega \mathrm{SST}^{(31)}$ models. Additional comparisons were made with other, simpler, models but these proved to be less effective and in order to remain concise these are not detailed here (but may be found in literature, ${ }^{(27)}$ ). For all results shown using the realisable $k-\varepsilon$ model, an enhanced wall model was used. A single operating condition for the wing and wheel combination was selected; the wing span, ride height, angle-of-attack, wheel track, and wheel width were chosen to be $S / c=1 \cdot 40, h / c=0 \cdot 13,8^{\circ}, T / c=1 \cdot 60 c$, and $W / c=0 \cdot 63 c$ respectively. The planes on which the comparisons were made are as depicted in Fig. 2, and the description here is extended to familiarise the reader with the details of the flow features to be analysed further in section 3 .

Using the freestream velocity as the reference velocity, normalised $x$-velocity, in-plane velocity vectors and turbulence intensity were used to compare each model with experimental LDA results. Indicative comparisons are presented here, drawn from an exhaustive set on all planes ${ }^{(27)}$. For station A, between the wing and wheel $(x / c=-0.63)$, qualitatively, each model predicts the expected $x$-velocity deficit in front and approximately mid-height of the wheel as well as forward and above the contact patch region (Fig. 6). The first of these two regions, located at $z / c=0 \cdot 6, y / c=1 \cdot 3$, indicates the stagnation point at the most upstream location of the wheel. The second region, at $z / c=0 \cdot 2, y / c=1 \cdot 3$, indicates the location at which the main wing vortex passes through the $x / c=-0.63$ plane. This vortex is formed by the pressure difference about the bottom inboard edge of the wing's endplate (indicated by the grey outline). The local normalised $x$-velocity component in this region due to this flow structure was measured as a normalised value of approximately $-0 \cdot 2$. Additionally, the formation of a secondary wing vortex generated from the pressure differential across the top edge of the endplate is also evidenced at $y / c=1 \cdot 5, z / c=0 \cdot 6$ by a third, smaller region where the $x$-velocity reduces to a value of $0 \cdot 2$. Again, both turbulence models indicate a similar velocity deficit and location for both structures. 

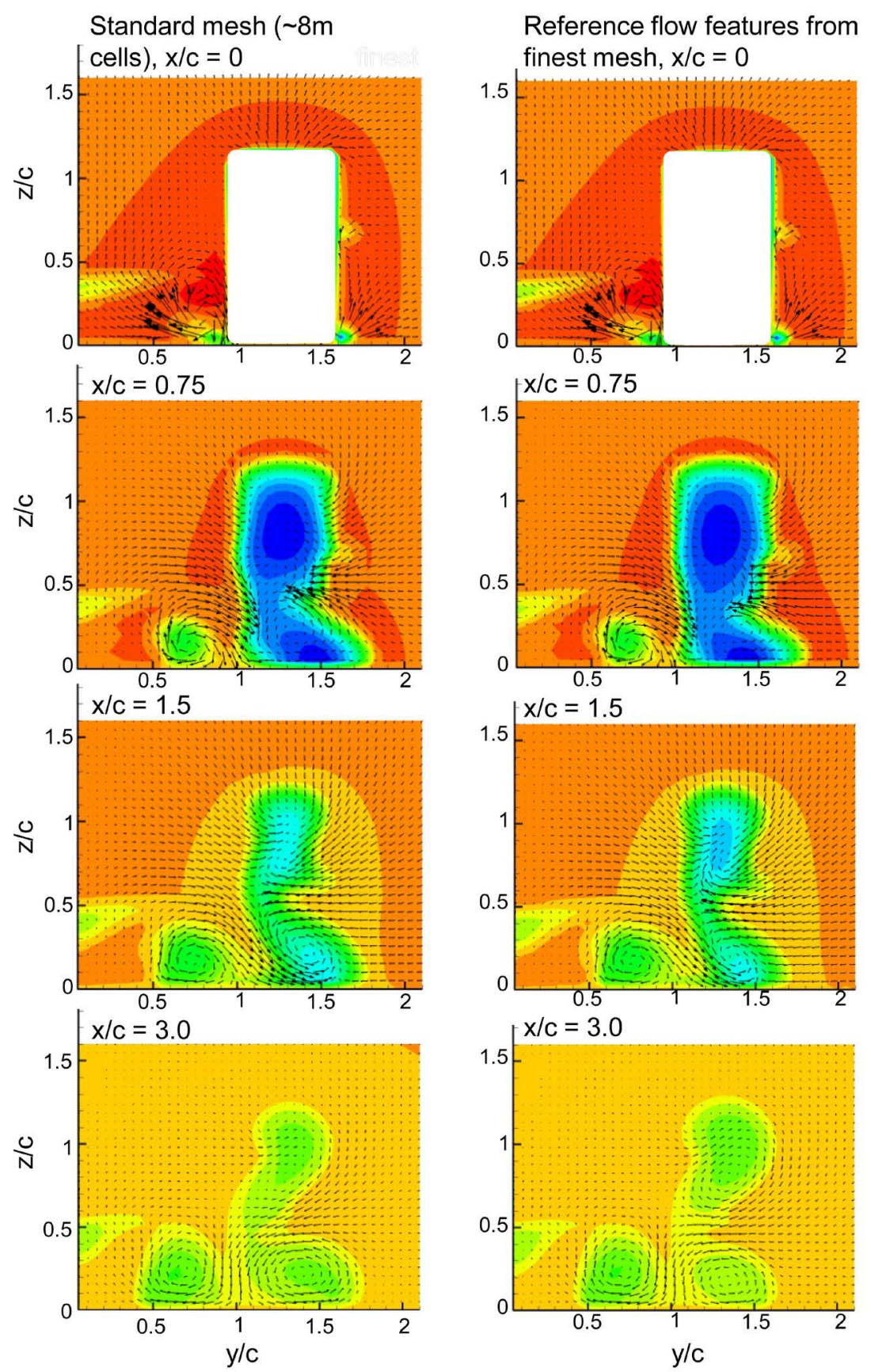

Normalised $x$-velocity

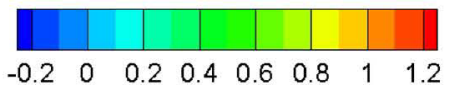

Figure 5 . Variations in velocity magnitude between the chosen mesh and the finest one tested, on planes at $x / c=0,0.75,1.5$ and 3.0 . 
(a) Experimental LDA results
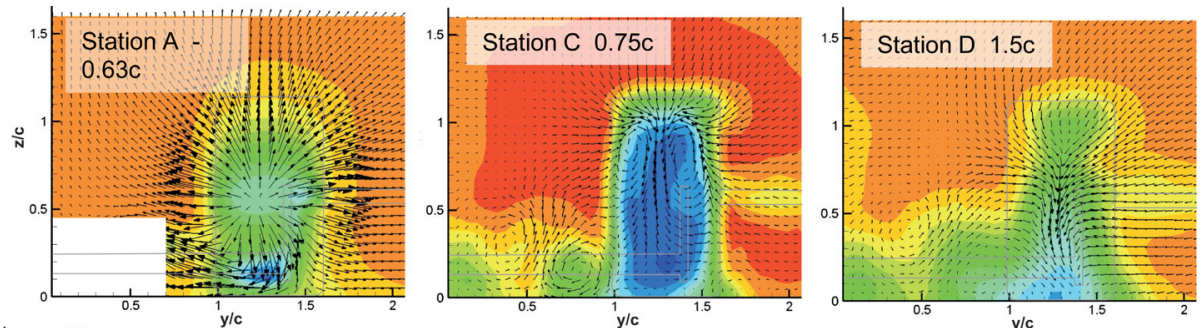

(b) $k-\omega \mathrm{SST}$
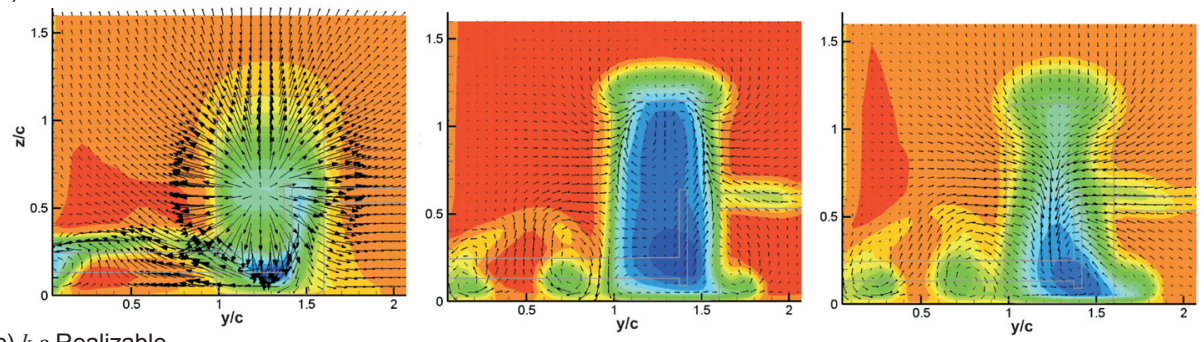

(c) $k$ - $\varepsilon$ Realizable
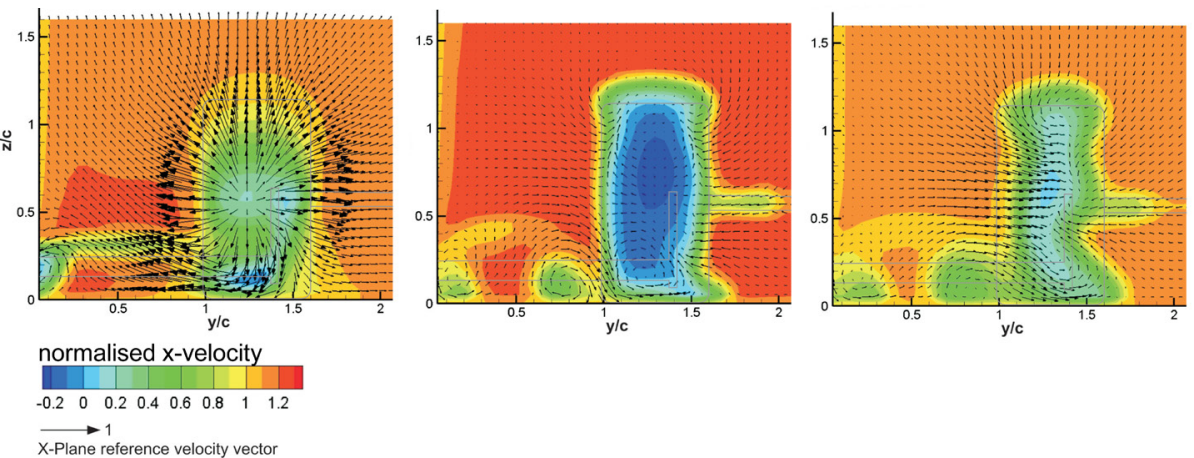

Figure 6. Normalised $x$-velocity and in-plane comparisons to LDA results at $x / c=-0.63$ (station A), $x / c=0.75$ (station $C$ ), and $x / c=1.5 c$ (station $D)$ for different turbulence models.

Behind the wing, a strong cross-component velocity is seen adjacent to the ground in the region of $0.7<y / c<1 \cdot 3$, caused by the suction generated beneath the wing and the subsequent upwash behind it. The extent of the inboard and vertical components of the cross flow is consistent across all results. The experimental results indicate that the peak turbulence intensity occurs inboard of the main wing vortex core $(y / c=1 \cdot 3, z / c=0 \cdot 2)$. A local increase is also seen at the core of the secondary wing vortex $(y / c=1 \cdot 55, z / c=0 \cdot 55)$, but other than in the wake of the wing $(0 \cdot 7<y / c<$ $1)$, no other regions of significantly increased turbulence were recorded. Both turbulence models indicate increased turbulence intensity at the core of the main wing vortex, but the $k$ - $\varepsilon$ realisable model most accurately predicts the magnitude in this region (Fig. 7) with a variation of approximately $10 \%$ in comparison to the LDA measurements.

The local maximum obtained at the core of the secondary wing vortex was also predicted by both turbulence models, with $k-\omega$ SST determining this feature most accurately with a turbulence intensity variation of approximately $5 \%$ to the experimental measurement (Fig. 7(d)). This was the only region where the $k-\varepsilon$ realisable model was outperformed. Unfortunately, both, but more 
(a) Experimental LDA results
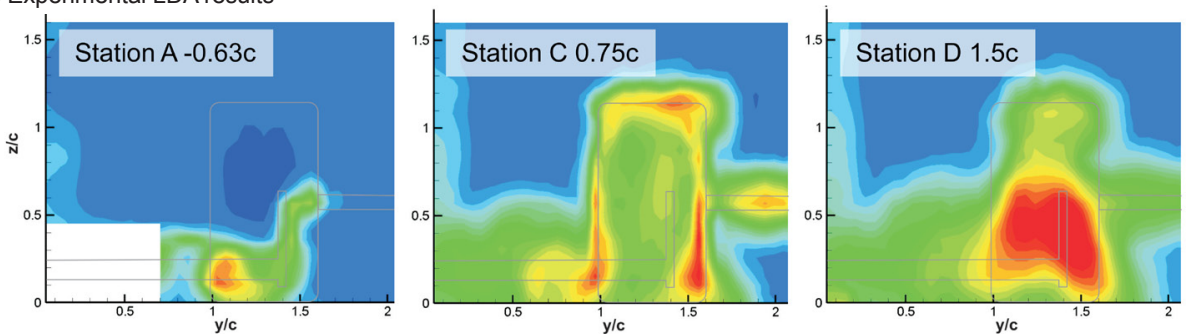

(b) $k-\omega \mathrm{SST}$
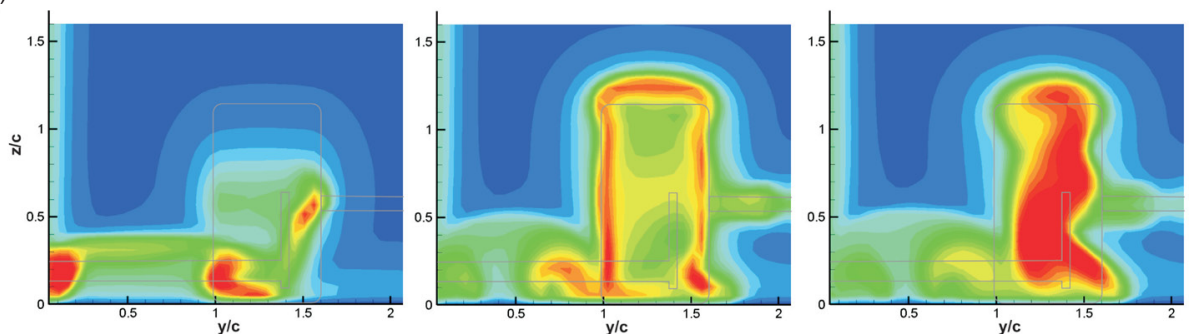

(c) $k-\varepsilon$ Realizable
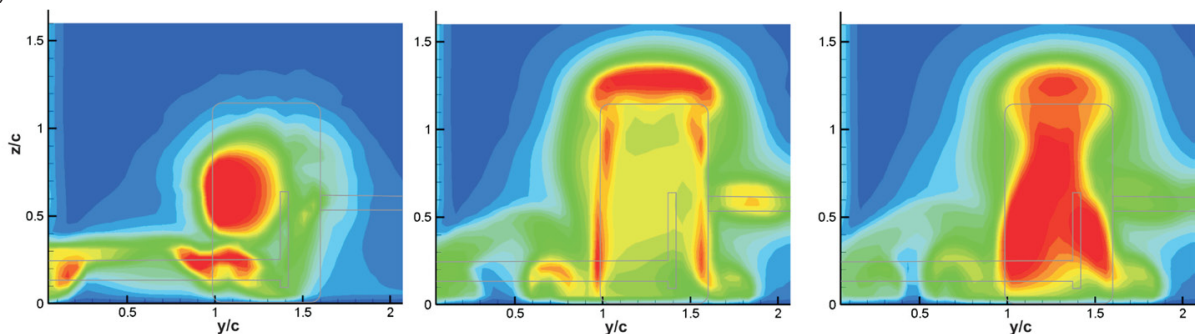

turbulent intensity

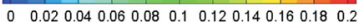

Figure 7. Turbulence intensity comparisons to LDA results at $x / c=-0.63$ (station $A$ ), $x / c=0.75$ (station $C$ ), and $x / c=1.5 c$ (station $D$ ) for different turbulence models.

so the SST, models calculated a large - and potentially non-physical - increase in turbulence intensity forward of the wheel corresponding to the location of the expected stagnation region.

On the plane located at $x / c=1 \cdot 5$, the experimental results indicated that the upper wheel wake vortices had dissipated, and this region of the wheel wake is mostly dominated by the downwash component $(y / c=1 \cdot 3,0 \cdot 3<z / c<0 \cdot 8)$ which was first observed on the Station C (Fig. 6.). Without the upper wheel wake vortices, the flow entrainment into the wheel wake is no longer solely from the top centre, but now also includes the sides, best captured by the $k$ - $\omega$ SST model. The experimental results also indicate that the height of the wheel wake remains the same and that the upper region progresses outboard. The general height and position of the upper wheel wake has been captured best by the $k-\varepsilon$ realisable model (Fig. 6). The $k$ - $\omega$ SST model maintains the height of the wheel wake from the previous plane, but indicates no variation in the horizontal position. At the base of the wheel wake, like the experimental results, the lower wheel wake in both models broadens and, on the inboard region, encroaches that of the wing wake. The lower wheel wake of the $k$ - $\omega$ SST model now also includes a lower vortex structure (Fig. $7 f, y / c=1 \cdot 4, z / c=0 \cdot 2$ ) that was previously observed with the realisable model on the $x / c=0.75$ plane. 
The $k$ - $\varepsilon$ realisable model outperforms the $k-\omega$ SST model in the vicinity of the wheel wake, with a better prediction of the $x$-velocity deficit in this region. Reductions in the surrounding cross component vectors that define the main wing vortex indicate that the strength of this vortex has been reduced. Similarly, the wing and wall junction vortex also reduces in strength, but the core is now located approximately $0 \cdot 1 c$ higher $(y / c=0 \cdot 4, z / c=0 \cdot 4)$. The reduction in strength and the variation in the direction of the subsequent flow between these two vortices was best predicted by the realisable model.

Both models indicate that the base of the wake in general contains higher values of turbulence intensity than that of the upper wheel wake region (Fig. 7); in the upper region of the wheel wake it is over-predicted by both. Despite this, the $k$ - $\varepsilon$ realisable prediction is marginally better than the $k$ - $\omega$ SST, given that the upper wheel wake region is smaller. The realisable model also indicates that the outboard side of the wheel wakes base should have larger I values than the inboard side.

It was decided to continue the simulations using solely the $k-\varepsilon$ realisable turbulence model. While neither of the turbulence models proved definitively suitable for the complexities of the flowfield, this was considered to be the best compromise, most-accurately predicting the size, and velocity deficit, of the wheel wake indicating a superiority at predicting the separation point from the wheel tread and side walls. The $k$ - $\varepsilon$ realisable model also best predicted the flow structures associated with the wing wake, particularly the main wing vortex shape and position.

Additionally the, turbulence intensity comparison indicated that the variation in the velocities calculated with the $k-\varepsilon$ realisable model were the most consistent with the LDA data, and significantly better than the $k$ - $\omega$ SST predictions. The choice of model is in agreement with other studies in literature which also concluded effectiveness for both wheels and inverted wings ${ }^{(5,32,33)}$.

\subsection{NUMERICAL RESULTS AT FULL SCALE}

From the full parametric spread of results obtained at all angles of attack, spans, and ground clearances, three primary types of interaction were identified, as shown in Fig. 8. In order to explore the causes and consequences of these, the subsequent analysis examines three cases that are representative of the extreme states.

With $0^{\circ}$ angle-of-attack and a span equal to the wheel track, both wing vortices where able to travel around the outer face of the wheel (Fig. 8, interaction A). From this configuration, increasing the angle-of-attack to $12^{\circ}$ caused the main wing vortex to travel inboard of the wheel (interaction B) while the secondary vortex retained an outside path. Reducing the span of the wing such that the endplate was in line with the inner face of the wheel resulted in the secondary vortex also travelling along the inside face of the wheel (interaction $\mathrm{C}$ ).

Figure 9 indicates the variations in lift and drag for each of the examples depicted in Fig. 8 in comparison to the same wing in isolation, and the lift and drag of the wheel when in combination with the three different wing configurations and also in isolation. In these figures, the lift and drag coefficient areas are presented since the reference area of the wing would vary with the span and it is desirable to obtain an understanding of the relative variation in negative lift (downforce) and drag magnitudes for each case.

Interaction A, with a wing span and angle-of-attack of $1.60 c$ and $0^{\circ}$ respectively, exhibited approximately a 50\% and 70\% reduction in downforce and drag respectively while interaction $\mathrm{B}$, with the same span but an angle-of-attack of $12^{\circ}$, showed less significant reductions in negative lift and drag of $12 \%$ and $24 \%$ respectively. Interaction C showed an opposite trend, with the wing in the presence of a wheel increasing in downforce and drag by $10 \%$ and $22 \%$ respectively. 
(a)

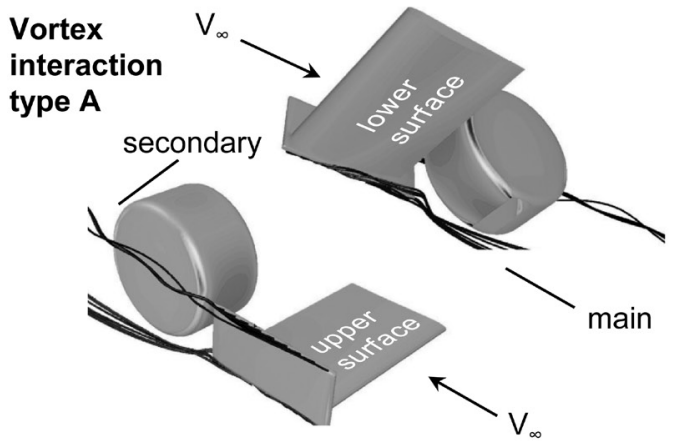

(both vortices travel outboard of wheel)

(b)

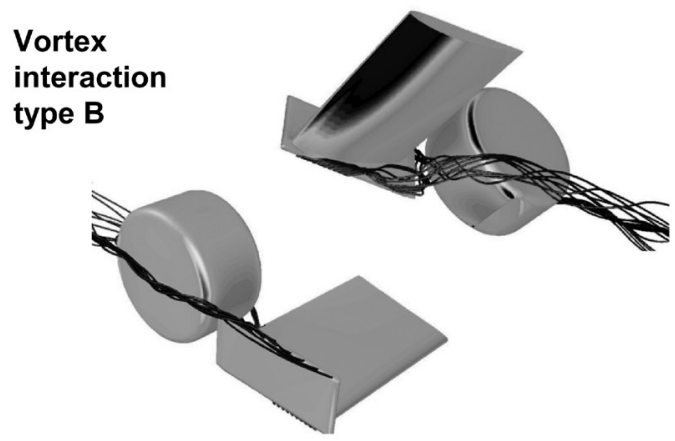

(main travels inboard, secondary travels outboard)

(c)
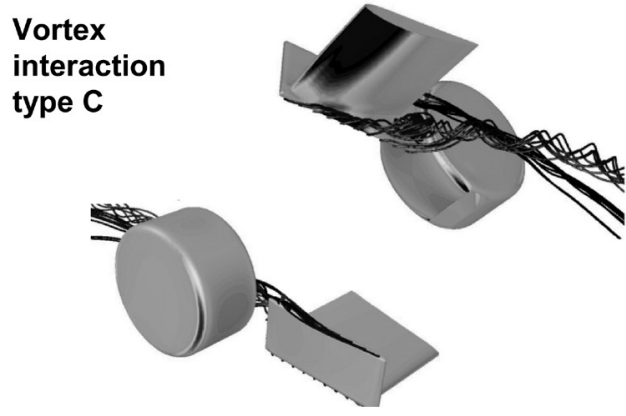

(both vortices travel inboard of wheel)

Figure 8. The three wing and wheel vortex interactions;

(a) both outboard, (b) main inboard and secondary outboard, and (c) both inboard.

The variation of the wheel forces relative to the isolated case also differed for each configuration. With the wing at an angle-of-attack and span of $0^{\circ}$ and $1.6 \mathrm{c}$ respectively, the wheel experienced no significant change in lift and drag. Increasing the angle-of-attack to $12^{\circ}$ resulted in both the lift and drag of the wheel reducing to $45 \%$ and $28 \%$ of the isolated case. Reducing the wing span to $0.97 \mathrm{c}$ increased both the lift and drag of the wheel such that drag was equal to the isolated case, and lift was $25 \%$ less. 
(a)

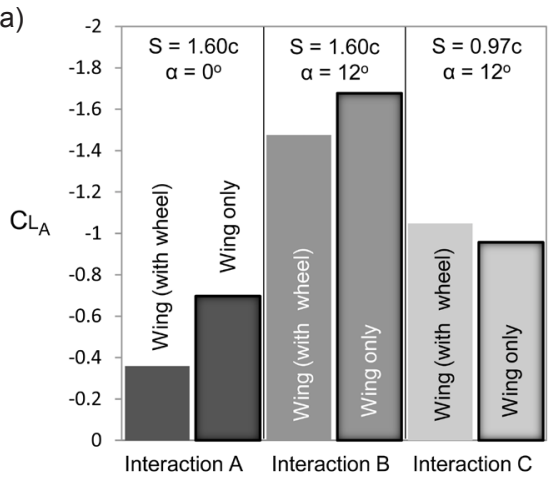

(c)

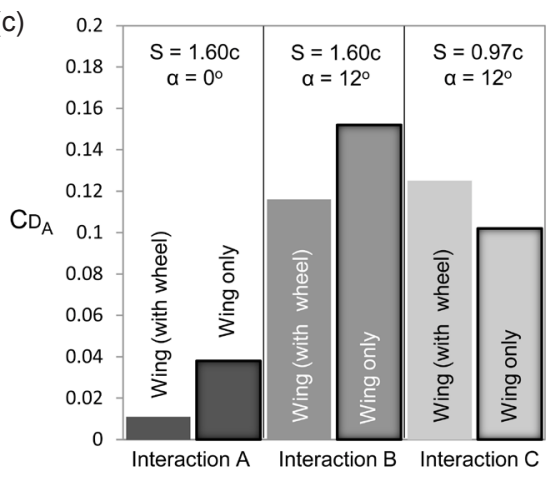

(b)
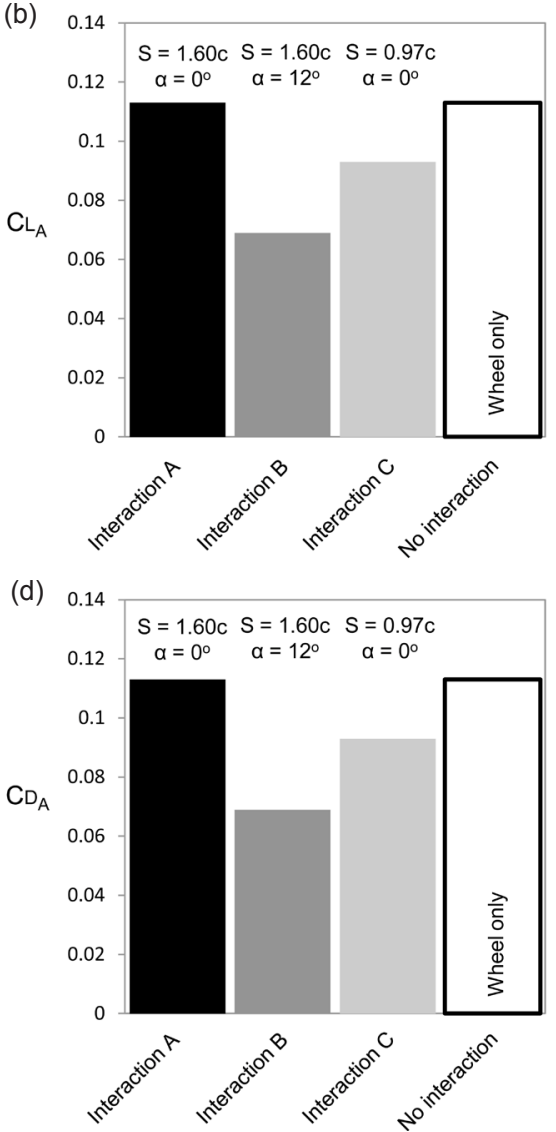

Figure 9. (a) wing lift coefficient, (b) wheel lift coefficient, (c) wing drag coefficient, (d) wheel drag coefficient for different interaction states.

\subsection{Causes and consequences of both wing vortices travelling outboard of wheel (interaction A)}

Normalised $x$-vorticity and in-plane vectors are shown in Fig. 10 for $x$ planes located between the wing and wheel $(x / c=-0.63 c)$ and at the wheel centre $(x / c=0)$ for interaction A. For reference, the projected outlines of the wing and wheel have also being included. From the results of the isolated wing case, it is evident that the main wing vortex is approximately four times stronger than that of the secondary vortex (Fig. 10(a). Moving downstream to the $x / c=0$ plane (Fig. 10(b)), the main vortex travels $0 \cdot 2 c$ inboard and slightly higher, while the secondary vortex moves outboard and lower.

Considering the $x / c=-0.63$ plane for the pair of isolated wheels (Fig. 10(c)), the vectors demonstrate that the stagnation point forward of each wheel can generate cross flow components up to a value of 0.7 times that of the free stream velocity, $U_{\infty}$. Similarly, regions of cross flow created by the ejected flow from the either side of the contact patch (Fig. 10(d)) reached magnitudes of $0 \cdot 6 U_{\infty}$. It is apparent that the presence of the wheel alters the formation and direction of the main wing vortex (Fig. 10(e)). The outboard cross flow component created by the large pressure rise associated with the viscous jetting action forward of a rotating wheel (evident on the $x / c=-0.63$ 
(a) Isolated wing, $x / c=-0.63$

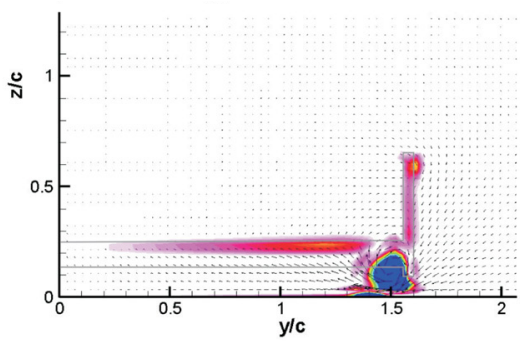

(c) Isolated wheel, $x / c=-0.63$

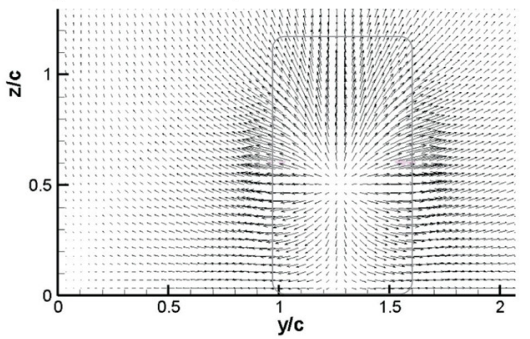

(e) Combined wing and wheel, $x / c=-0.63$

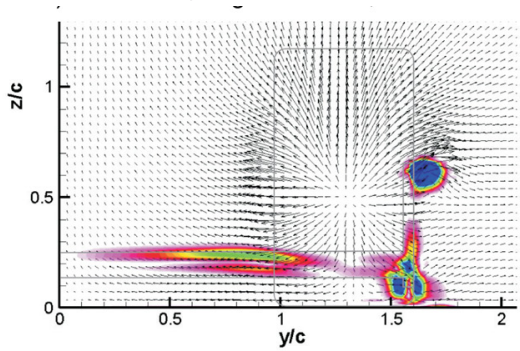

(b) Isolated wing, $x / c=0$

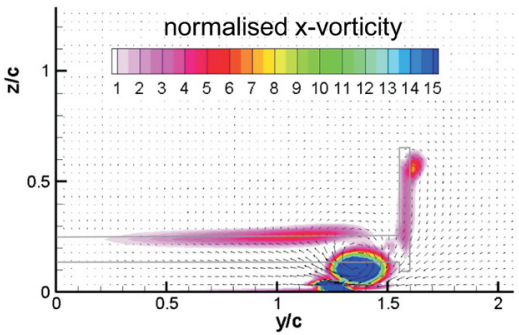

(d) Isolated wheel, $x / c=0$

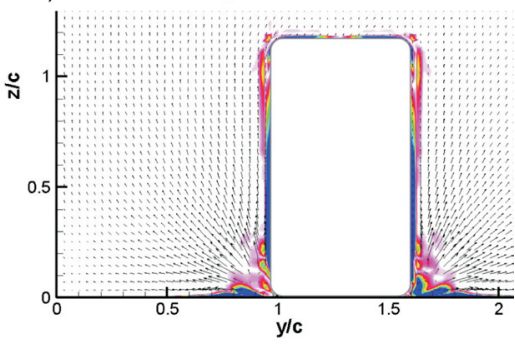

(f) Isolated wing and wheel, $x / c=0$

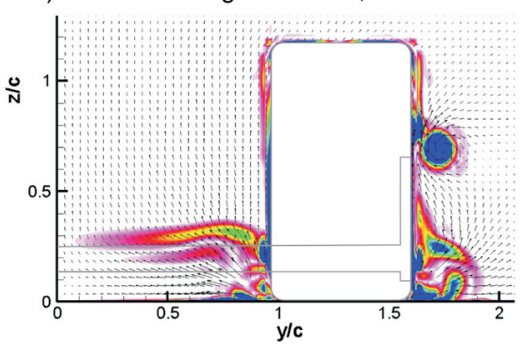

Figure 10. Vectors and Vorticity on $x$-planes at stations $A$ and $B$ for a wing and/or wheel with $W / c=0.63, T / c=1 \cdot 6, h / c=0 \cdot 13, S / c=1 \cdot 6 c$ and $A O A=0^{\circ}$ (vortex/interaction type A).

plane) remains. This forces the main wing vortex, which is now approximately half the strength of that for the isolated case, outboard of the wheel (Fig. 10(f)).

The reduction in the main wing vortex strength is consistent with previous reports linking the downforce produced by an isolated wing to the strength of the wing's main vortex ${ }^{(3)}$, but an additional variation in the main wing vortex structure also exists. While this vortex for the isolated case is round and completely coherent (Fig. 10(a)), the main wing vortex for the combined wing and wheel appears to consist of three individual components (Fig. 10(e), $y / c=1 \cdot 6, z / c=0 \cdot 15$ ). The main wing vortex forms from the variation in pressure that occurs across the bottom edge of the endplate.

Figure 11 shows pressure coefficients on planes coincident with the upper and lower edges of the front wing endplate. For an isolated wing, pressure is consistently lower on the inboard section of the endplate in comparison to the pressure outboard of the endplate $(-1 \cdot 85<x / c<-0 \cdot 85, y / c>$ $1 \cdot 6)$; For the combined wing and wheel (Fig. 11(a)), this pressure difference varies along the length of the endplate such that at the front of the endplate $(-1 \cdot 85<x / c<-1 \cdot 1, y / c=1 \cdot 6)$ it is determined by the suction beneath the wing while at the rear $(-1 \cdot 1<x / c<-0 \cdot 85, y / c=1 \cdot 6)$, the rise created forward of the wheel causes an opposite pressure variation. This inconsistent variation along the 
Combining wing and wheel, $z / c=0 \cdot 1$
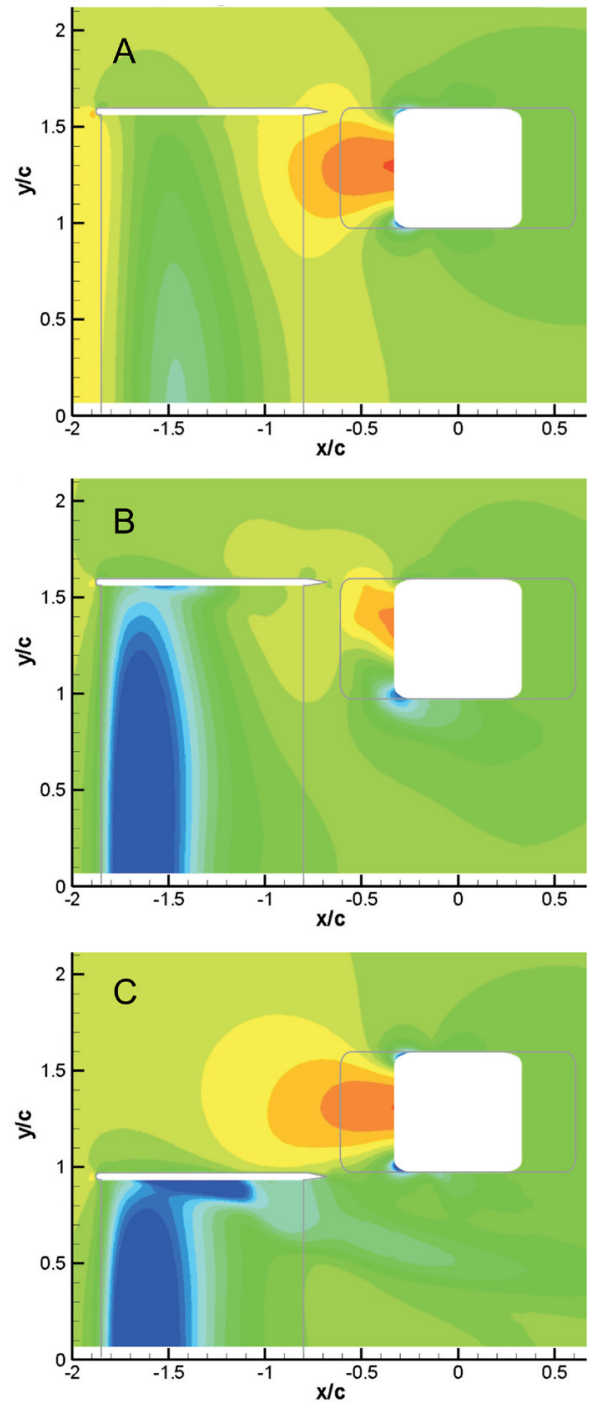

Combining wing and wheel, $z / c=0.59$
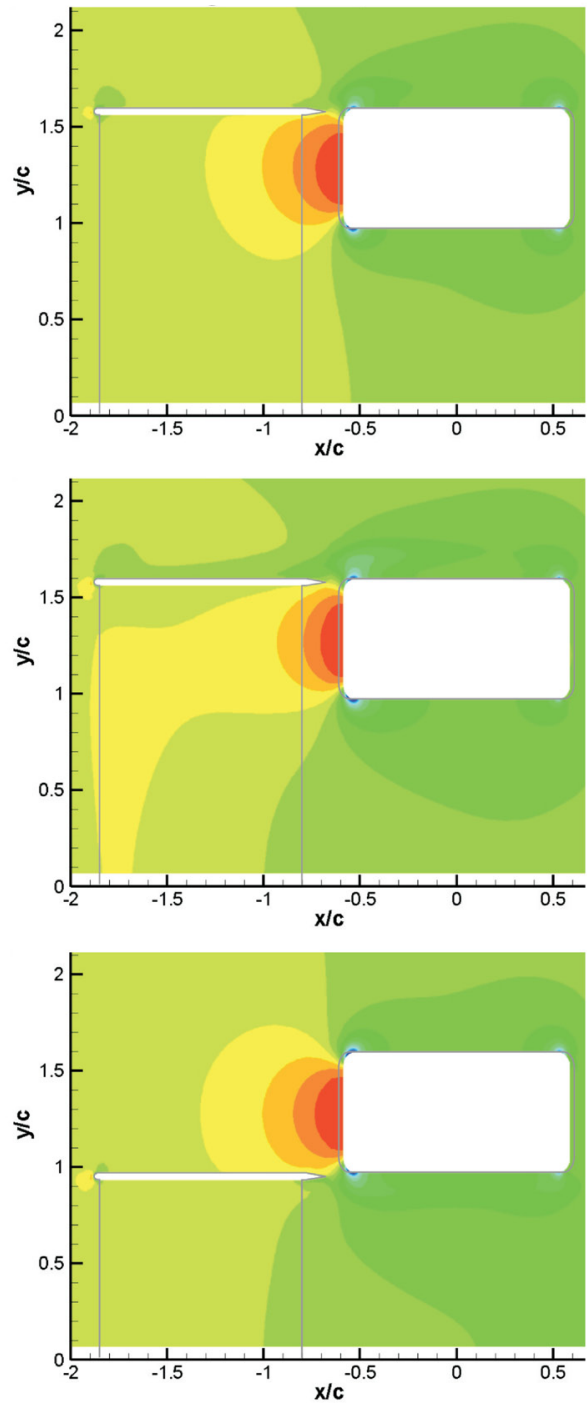

pressure coefficient

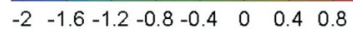

Figure 11. Pressure coefficients around endplate on planes $z / c=0.1$ and 0.59 for a wing and/or wheel with vortex/interaction type A, B and C.

length of the endplate causes the vortex that sheds from the bottom edge of the endplate to be less coherent in the presence of the wheels.

In the presence of the wheel, the upper vortex is three times stronger than the main wing vortex (Fig. 10(e,f)) and more than three times the isolated wing vortex (Fig. 10(a,b)). The pressure variation for the combined wing and wheel (Fig. 10(f)) case increases due to the presence of the stagnation region formed at the most upstream location of the wheel $(x / c=0 \cdot 45, y / c=1 \cdot 3)$. This stagnation region for the combined wing and wheel is unaffected by the presence of the wing 
(a) Isolated wing, $x / c=0.75$

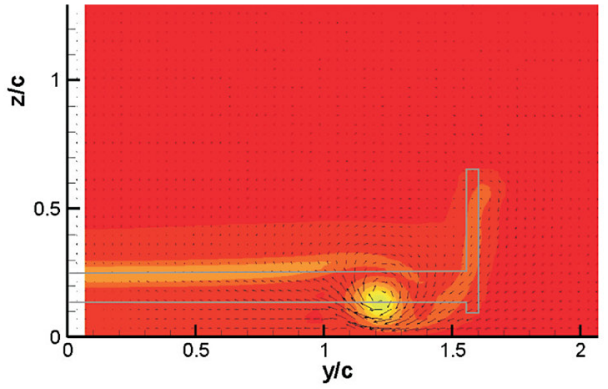

(c) Isolated wheel, $x / c=0.75$

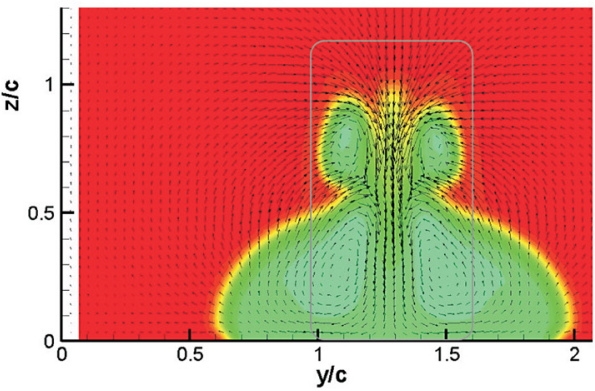

(e) Combined wing and wheel, $x / c=-0.75$

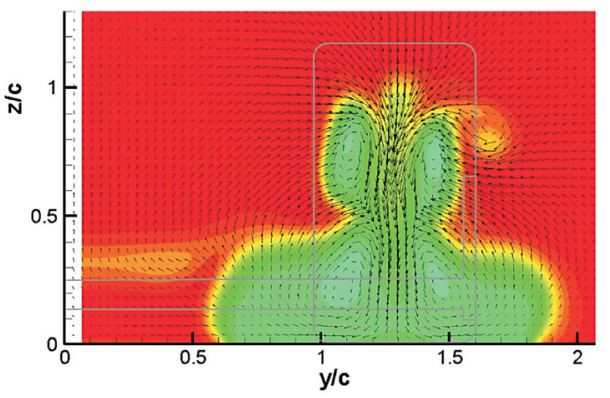

(b) Isolated wing, $x / c=3$

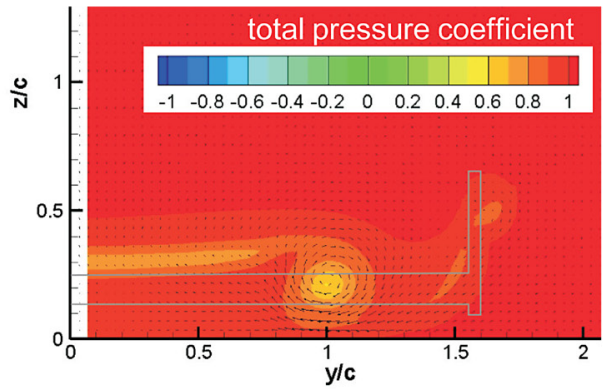

(d) Isolated wheel, $x / c=3$

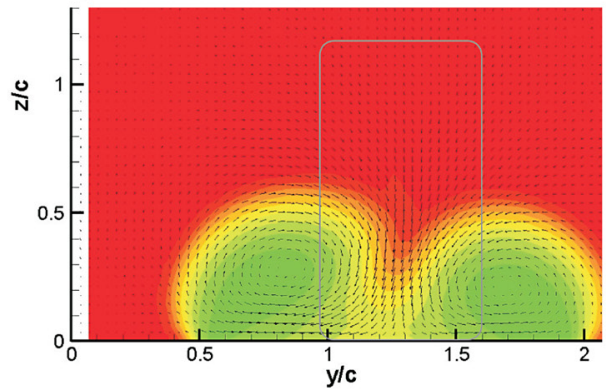

(f) Isolated wing and wheel, $x / c=3$

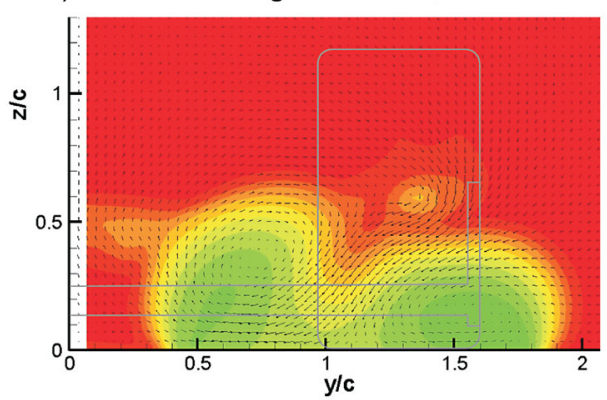

Figure 12. Vectors and total pressure on $x$-planes for a wing and/or wheel with $W / c=0.63$, $T / c=1.6, h / c=0.13, S / c=1.6 \mathrm{c}$ and $\mathrm{AOA}=0^{\circ}$ (vortex/interaction type A).

(Fig. 11(f)) and continues to produce large crossflow components (Fig. 10(e)) equal to that obtained for the isolated case (Fig. 10(c)).

The crossflow produced by the wheel interacts with the upper edge of the endplate to shed a stronger secondary vortex. Total pressure planes located at $x / c=0.75$ and $x / c=3$ indicate that the secondary vortex passes the outer side of the wheel cleanly (Fig. 12(e), $y / c=1 \cdot 65, z / c=0 \cdot 85$ ) and is then entrained into the upper wheel wake (Fig. 12(f) $y / c=1 \cdot 65, z / c=0 \cdot 85$ ). The increased strength of the secondary vortex also appears to allow it to travel further downstream than the same vortex for the isolated wing (Fig. 12(a,b). Total pressure contours plotted on planes located downstream from the wheel centre also indicate that the upper wheel wake becomes lower and wider for this interaction (Fig. 12(e)) relative to the wake belonging to the same isolated wheel (Fig. 12(c)). These variations in the wake structure indicate that the separation point from the upper 
wheel tread is further reward due to the wing. Despite this rearward shift in the separation point, no significant variation in lift and drag was obtained for the wheel for this interaction.

The suction pressure of the wing also acts on the wheel to reduce the static pressure experienced by the most upstream portion of the wheel tread and near the contact patch. This variation is likely to be reducing the lift and drag of the wheel and counter balancing the lift and drag increase expected by the delayed separation from the upper wheel. For this reason no variation is observed for the total forces experienced by the wheel due to the presence of the wing.

Downstream at the $x / c=$ three plane, the wheel wake produced by the combined wing and wheel (Fig. 12(f)) appears more asymmetric and travels closer towards the symmetry plane relative to the isolated wheel result (Fig. 12(d)). This was caused by the increased inboard component due to the pressure field from the wing ahead of the wheel. This is evident when comparing the vectors located in front of the wheel on the $x / c=-0.63$ plane for the isolated wheel and the combined wing and wheel (Fig. 10(c,e)). The increased crossflow observed at the inboard section of the wheel increases the separation along the inner face, and therefore increases the strength of the vortex that forms rearward of the wheel as a consequence. Accordingly, the inner wheel vortex is now larger than the outer wheel vortex, and draws the outer wheel vortex inboard as they both travel downstream.

\subsection{Causes and consequences of main wing vortex travelling inboard while secondary vortex travels outboard of wheel (interaction B)}

Increasing the wing angle-of-attack from $0^{\circ}$ to $12^{\circ}$, while maintaining the remaining wing and wheel parameters, caused the main wing vortex to travel inboard of the wheel while the secondary vortex remained outboard of the wheel (Fig. 8). The increase in angle-of-attack of the wing increased the suction beneath the wing (Fig. 11(b) and subsequently the strength of the main wing vortex and the flow entrainment towards the symmetry plane downstream of the wing (Fig. 13(c,d)). Comparing the main wing vortex size and position for the isolated wing case with an angle-of-attack of $0^{\circ}$ (Fig. 10(b)) to that of the $12^{\circ}$ case (Fig. 13(b)), the vortex is now almost twice the strength and the core has moved from $y / c=1.5$ to $y / c=1.35$ on the $x / c=-0.63$ plane, and from $y / c=1.35$ to $y / c=1.2$ for the $x / c=0$ plane.

The variation in position of the main wing vortex, relative to the peak pressure region created forward of the contact patch is the main cause for the main wing vortex travelling inboard of the wheel. In order to take this path, the main wing vortex is drawn across the face of the wheel and the vortex shape on the $x / c=0$ plane downstream for the wing in isolation compared to that of the wing in the presence of the wheel indicates that the main wing vortex structure is affected. This is due to the vortex being trapped between the converging ground and wheel surfaces, 'squashed' as a result.

At $x / c=-0 \cdot 63$, the main wing vortex for the isolated wing appears less coherent than that observed for the combined wing and wheel, potentially 'bursting' as previously described by Zerihan and Zhang ${ }^{(6)}$, suggesting that the wing is beneath the height at which the maximum downforce can be obtained. In the presence of the wheel, the wing produced $12 \%$ less downforce and the vortex structure suggests that the wing is able to operate closer to the ground without reducing downforce - something that will be investigated in a future study. A similar trend was observed during a previous investigation relating to the effect that varying the span has on the downforce loss phenomenon ${ }^{(6)}$. The shorter aspect ratio wing produced less total downforce and subsequently suffered from a less severe adverse pressure gradient in the centre of the wing; comparing the suction generated by the wing in isolation and in the presence of a wheel provides an indication that the same phenomenon occurs here. 
The cause for the $12 \%$ reduction in downforce appears to be the same as that described for interaction 'A'. The low pressure region beneath the wing has been adversely affected by the high pressure region generated forward of the wheel, with the only variation being the extent to which the high pressure region forward of the wheel is affected by the wing suction. This more significant reduction in pressure contributes to the $26 \%$ reduction in lift for the wheel in this configuration. Unlike interaction ' $A$ ', the reduction in the high pressure region forward of the wheel (Fig. 11(b)) has ensured that the static pressure remains reduced inboard of the endplate $(-1.85<x / c<-0.85$, $y / c<1.57)$ in comparison to that experienced outboard along the entire endplate length $(-1.85<x / c$ $<-0.85, y / c<1.57)$, allowing a more coherent vortex structure to form. Additionally, the location of the maximum pressure forward of the wheel has shifted from $y / c=1.25$ for the isolated wheel to $y / c=1.4$ for the combined wing and wheel.

The size and strength of the secondary wing vortex is similar between interactions A and $\mathrm{B}$. In both interactions, the secondary vortices are essentially of equal strength, size, and position - and are rotating in the same direction as the main wing vortex. This is despite the isolated wing with an angle-of-attack of $12^{\circ}$ having a secondary vortex twice the strength of that obtained for the isolated wing with an angle-of-attack of $0^{\circ}$. The variation in the secondary vortex in the isolated condition is due to the pressure increase obtained on the top surface of the wing when the angleof-attack is increased.

Total pressure contour plots downstream indicate that the secondary vortex maintains the same position for interaction B (Fig. 14(c,d)) as for A (Fig. 12(e,f)). The most significant variation in the wake structure of this configuration, in comparison to that of the isolated wheel, was determined to be an increase in height and reduction in width. This change in the combined wing and wheel wake is the opposite of that obtained for interaction A and is caused by the additional upwash seen by the increase in wing angle-of-attack, causing the wheel separation point to shift further forward. This is confirmed by the velocity vectors located above the wheel on the $x / c=0$ (Fig. 13(d)) plane

(a) Isolated wing, $x / c=0.63$

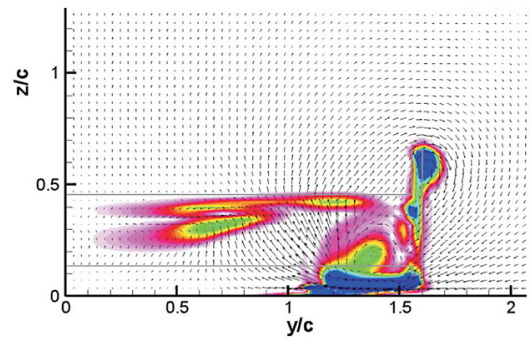

(c) Combined wing and wheel, $x / c=0.63$

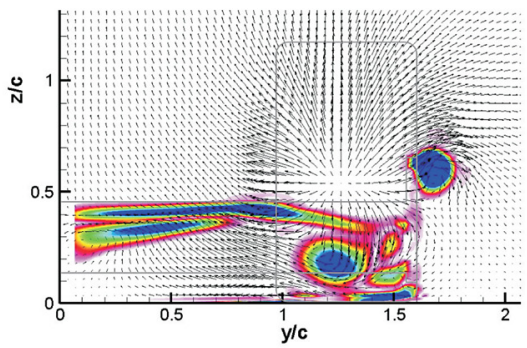

(b) Isolated wing, $x / c=0$

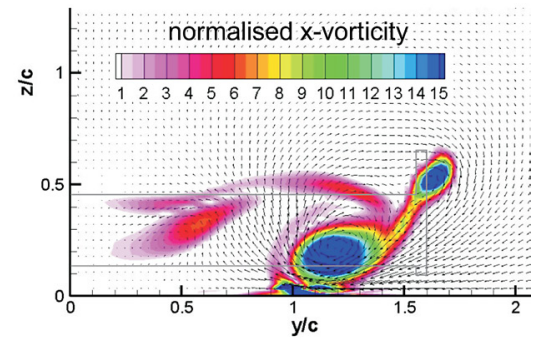

(d) Combined wing and wheel, $x / c=0$

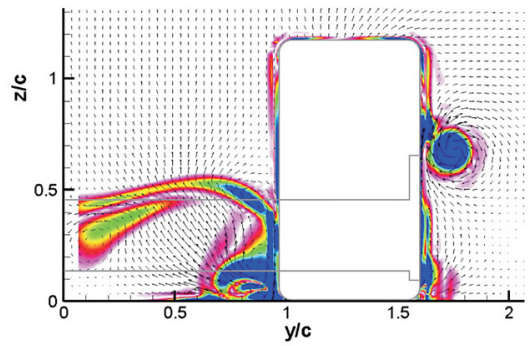

Figure 13. Vectors and vorticity on $x$-planes at stations $A$ and $B$ for a wing and/or wheel with $W / c=0.63, T / c=1.6, h / c=0 \cdot 13, S / c=1.6 c$ and $A O A=12^{\circ}$, (vortex/interaction type B). 
(a) Isolated wing, $x / c=0.75$

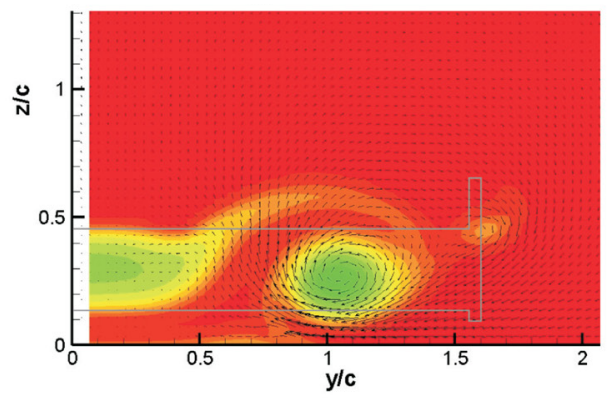

(c) Combined wing and wheel, $x / c=0.75$

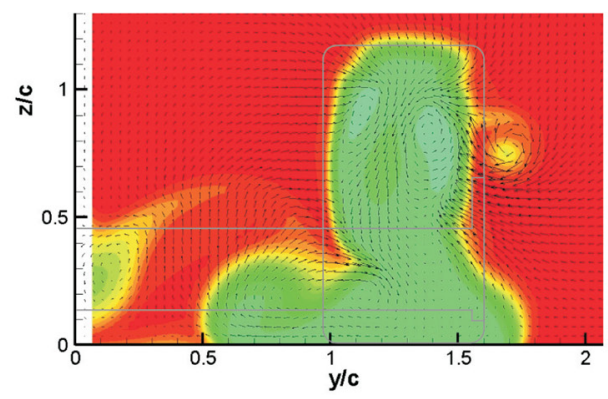

(b) Isolated wing, $x / c=3$

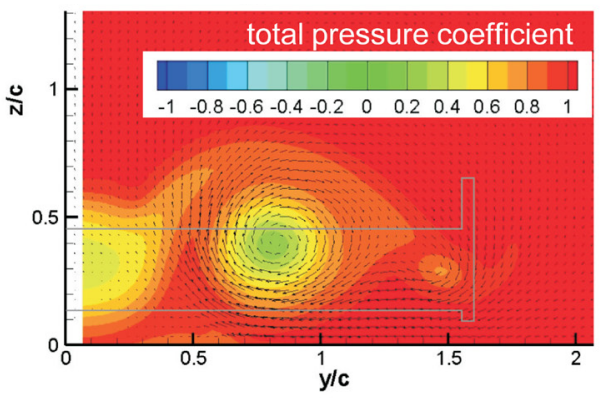

(d) Combined wing and wheel, $x / c=3$

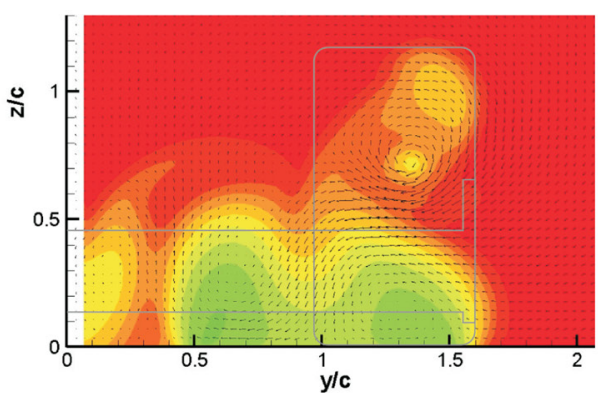

Figure 14. Vectors and total pressure on $x$-planes for a wing and/or wheel with $W / c=0 \cdot 63$, $T / c=1 \cdot 6, h / c=0 \cdot 13, S / c=1 \cdot 6 \mathrm{c}$ and $\mathrm{AOA}=12^{\circ}$ (vortex/interaction type B).

having a positive $\mathrm{z}$ velocity component four times greater than that obtained for the same isolated wheel (Fig. 10(d)). The $0 \cdot 2 c$ increase in wake height and the $28 \%$ reduction in drag for the wheel is consistent with the link between drag and the separation point. The variation in separation point would also be a significant contributor to the $45 \%$ reduction in negative lift obtained ${ }^{(27)}$.

\subsection{Causes and consequences of both wing vortices travelling inboard of wheel (interaction C)}

Reducing the span from $1.6 \mathrm{c}$ to $0 \cdot 97 \mathrm{c}$, such that the outer endplate aligns with the inner face of the wheel, allowed both the main and secondary vortices to travel inboard of the wheel (Fig. 8(c)). In interactions $\mathrm{A}$ and $\mathrm{B}$, and for all the isolated wing results, the secondary vortex always rotated in the same direction as the main wing vortex. For interaction $C$, the secondary vortex (Fig. 15(c), $y / c=0 \cdot 88, z / c=0.6$ ) rotates in the opposite direction of the main wing vortex (Fig. 15(c), $y / c=0.5$, $z / c=0 \cdot 2)$. While for both interaction A and B a stronger secondary vortex was caused due to the pressure rise provided by the stagnation region at the most upstream position of the wheel, static pressures on the $z / c=0.59$ plane for the combined wing and wheel (Fig. 11(c)) indicate that at this span, the endplate sits inboard of the high pressure region created by the same wheel stagnation region. Vectors located on the $x / c=-0.63$ plane confirm that the new endplate position relative to the wheel stagnation is the cause for the opposite vortex rotation (Fig. 15(d)).

This downforce increase again coincided with a consistent variation in size and strength of the main wing vortex. For interactions $\mathrm{A}$ and $\mathrm{B}$, the main wing vortex was reduced in strength by the presence of the high pressure region created at the front contact patch; interaction $\mathrm{C}$ yields an increase in main wing vortex strength $(y / c=0 \cdot 5, z / c=0 \cdot 2$, Fig. $15(d))$ over the same wing in 
(a) Isolated wing, $x / c=-0.63$

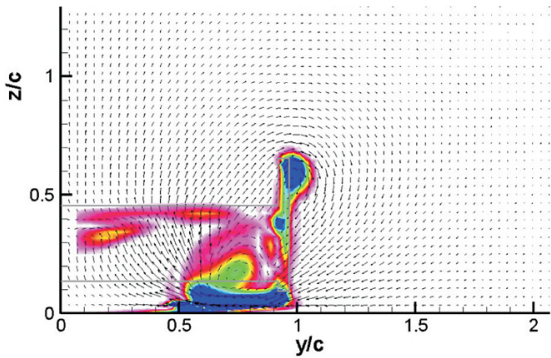

(c) Combined wing and wheel, $x / c=-0.63$

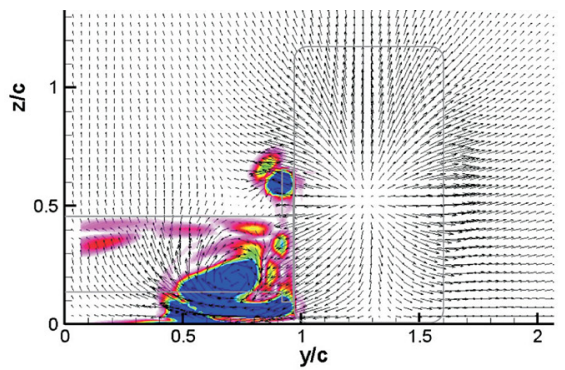

(b) Isolated wing, $x / c=0$

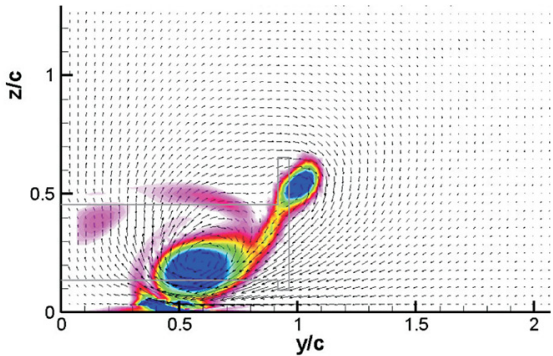

(d) Combined wing and wheel, $x / c=0$

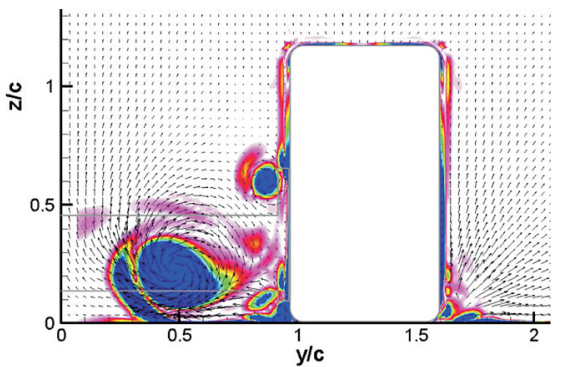

Figure 15. Vectors and vorticity on $x$-planes at stations $A$ and $B$ for a wing and/or wheel with $W / c=0 \cdot 63, T / c=1 \cdot 6, h / c=0 \cdot 13, S / c=1 \cdot 6 c$ and $A O A=12^{\circ}$, (vortex/interaction type C).

(a) Isolated wing, $x / c=0.75$

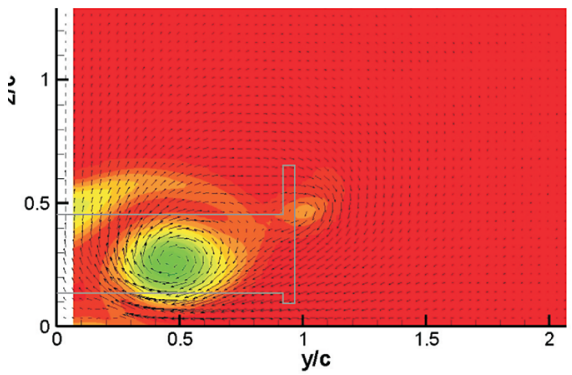

(c) Combined wing and wheel, $x / c=0.75$

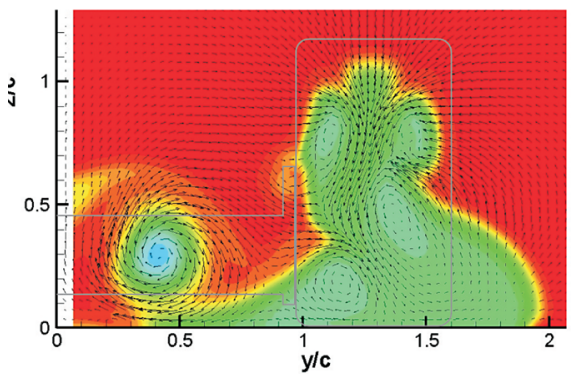

(b) Isolated wing, $x / c=3$

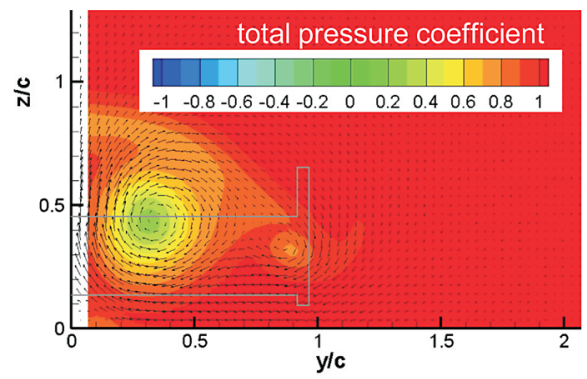

(d) Combined wing and wheel, $x / c=3$

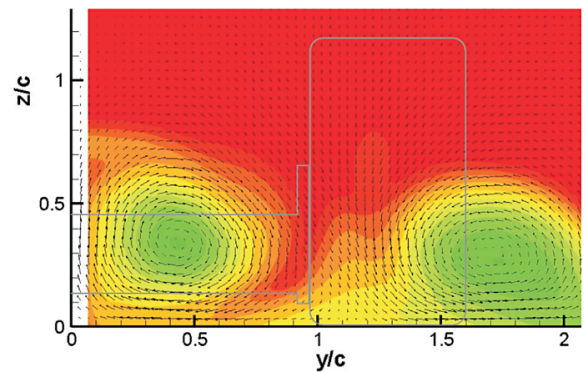

Figure 16. Vectors and total pressure on x-planes for a wing and/or wheel with $W / c=0.63$, $T / c=1 \cdot 6, h / c=0 \cdot 13, S / c=1 \cdot 6 c$ and $A O A=12^{\circ},($ vortex/interaction type $C)$. 
isolation $(y / c=0 \cdot 5, z / c=0 \cdot 2$, Fig. 15(b)) due to the same flow feature $(x / c=-0 \cdot 4, y / c=1 \cdot 3$, Fig. $11 c$ ). At this wing span, the high pressure from the wheel's contact patch assists with increasing the pressure difference on the bottom edge of the endplate $(-1.85<x / c<-0.85, y / c=0.95$, Fig. $11 c$ ), increasing the inboard y velocity component of the flow beneath the endplate $(y / c=0.95$, $z / c=0 \cdot 05$, Fig. 15(c)) and creating a stronger vortex $(y / c=0 \cdot 5, z / c=0 \cdot 2$, Fig. 15(d)).

The main wing vortex can also assist with reducing separation ${ }^{(6)}$. Total pressure contours on planes located at $x / c=0.75$ confirm that the separation experienced by the wing $(y / c=0 \cdot 1, z / c=$ $0 \cdot 5$, Fig. 16(a)) has been reduced in the presence of the wheel $(y / c=0 \cdot 1, z / c=0 \cdot 5$, Fig. 16(c)). The reduced separation from the bottom surface of the wing has enabled the flow volume drawn beneath the wing to increase and for this reason the suction generated by this wing in the presence of the wheel is greater than that experienced by the same wing in isolation. This is a contributing factor to the $10 \%$ increase in downforce for the wing, while the increased vortex strength would be expected to drive the $22 \%$ increase in wing drag.

Unlike in the previous interactions, the main wing vortex continues downstream of the wheel without combining with the wheel wake structure and is clearly defined on the $x / c=0.75$ $(y / c=0 \cdot 4, z / c=0 \cdot 3$, Fig. 15(c) $)$ and $x / c=3(y / c=0 \cdot 4, z / c=0 \cdot 4$, Fig. 15(d)) planes. Including the main wing vortex, six individual vortices are evident on the $x / c=0.75$ plane (Fig. 15(c)). These also include: the two main wheel vortices $(y / c=1 \cdot 15, z / c=0.2$ and $y / c=1 \cdot 35, z / c=0.45)$; the two upper wheel vortices $(y / c=1 \cdot 1, z / c=0.8$ and $y / c=1 \cdot 4, z / c=0 \cdot 8)$; and the decaying secondary wing vortex $(y / c=0 \cdot 95, z / c=0 \cdot 6)$. By the $x / c=3$ plane (Fig. 15(d)) only the main wing vortex $(y / c=0 \cdot 5, z / c=0 \cdot 4)$ and outer primary wheel vortex remain $(y / c=1 \cdot 7, z / c=0 \cdot 3)$. The inner wheel vortex strength has been reduced and subsequently decays prior to reaching the $x / c=3$ plane as a result of the interaction with the wing.

An additional variation observed for the wheel wake of the combined wing and wheel is the more inboard location and increased height of the upper wheel wake on the $x / c=0.75$ plane (Fig. 15(e)) relative to that experienced by the isolated wheel case. As stated previously, the higher position is likely to be due to a more forward separation point from the wheel tread as experienced by interaction $\mathrm{B}$ and can again be linked to the $25 \%$ lift reduction experienced by the wheel over the isolated case. The $0 \cdot 05 c$ inboard shift of the upper wheel wake may be explained by the position and rotation of the secondary wing vortex.

In summary, it appears that the angle-of-attack can control the transition from interaction A to $\mathrm{B}$ and that from either interaction, adjusting the span can lead to achieving interaction C. Points of transition from one state to the next will be explored in future work.

\subsection{CONCLUSIONS}

A study into the aerodynamics of an open-wheel racing car front wing and wheel arrangement was conducted using CFD. There are three main interactions that can occur depending on the path that the main and secondary wing vortices take around the wheel. For interaction A to occur, the endplate must be positioned outboard of the stagnation regions forward of the wheels. These high pressure regions force the main and secondary wing vortices outboard, and reduce the pressure difference experienced on the bottom surface of the endplate, causing a weakened main wing vortex. The wheel wake, lift and drag values were largely unaffected while the wing experienced a $50 \%$ and $70 \%$ reduction in downforce and drag respectively due to the low pressure surface of the wing being exposed to the high pressure regions generated forward of the wheel.

Interaction B was achieved by increasing the wing's angle-of-attack. The suction generated beneath the wing causes the main wing vortex to be drawn inboard, and to pass the wheel along 
its inner face while the secondary vortex remains outboard. In this configuration the wing's ability to generate low pressure still suffers due to the high pressure regions generated by the wheel and thus the wing produces $12 \%$ less downforce. These high pressure regions are weakened and translated outboard by the presence of the suction beneath the wing. When this interaction is achieved, the influence of the main wing vortex on the wheel wake and the upwash generated by the wing forward of the wheel both contribute to lift and drag reductions of approximately $45 \%$ and $25 \%$ respectively for the wheel.

Reducing the span of the wing to align with the inner face of the wheel invokes interaction $\mathrm{C}$. In this condition, the secondary vortex, rotating in the opposite direction to that of the previous two configurations, passes inboard of the wheel and the main vortex is strengthened. Both features are caused by the wheel stagnation region now being located outboard of the wing's endplate. The stronger main wing vortex allows the wing to generate up to $10 \%$ more downforce and $22 \%$ more drag, as it alleviates separation on the suction surface while increasing the induced drag. Wheel forces remained largely unchanged.

It is clear that both angle-of-attack and span are sensitive parameters for combined wing and wheel performance and careful optimisation is required to ensure the most effective and safe handling of the vehicle - locating the precise points at which transition between interaction states occurs, and examining the role that ground clearance plays in performance, will form the basis of future work.

\section{REFERENCES}

1. Katz, J. Aerodynamics of race cars, Annu Rev Fluid Mech, 2006, 38, pp 27-63.

2. Dominy, R.G. Aerodynamics of Grand Prix Car Proc Institution of Mechanical Engineers, 1992, 206, pp 267-274.

3. ZhANG, X. and ZeRIHAN, J. Off-surface aerodynamic measurements of a wing in ground effect, $J$ Aircr, 2003, 40, (4), pp 716-725.

4. Agathangelou, B. and Gascoyne, M. Aerodynamic Considerations of a Formula One Racing Car, 1998. SAE 980399.

5. DoIG, G. and BARBER, T. J. Considerations for numerical modeling of inverted wings in ground effect, AIAA J, 2011, 49, (10), pp 2330-2333.

6. Diasinos, S., BARBER, T.J. and Doig, G. Influence of wing span on the aerodynamics of wings in ground effect, Proceedings of the Institution of Mechanical Engineers, Part G: Journal of Aerospace Engineering, 2013, 227, (3), pp 569-573.

7. ZeriHAN, J. and Zhang, X. Aerodynamics of a single element wing in ground effect, J Aircr, 2000, 37, (6), pp 1058-1064.

8. Zerihan, J. and Zhang, X. Aerodynamics of Gurney flaps on a wing in ground effect, AIAA J, 2001, 39, (5), pp 772-780.

9. Zhang, X. and Zerihan, J. Aerodynamics of a double-element wing in ground effect, AIAA J, 2003,41, (6), pp 1007-1016.

10. Zhang, X. and Zerihan, J. Edge vortices of a double element wing in ground effect, J Aircr, 2004, 41, (5), pp 1127-1137.

11. FACKRELL, J.E. The aerodynamics of an isolated wheel rotating in contact with the ground (Doctoral dissertation, Imperial College London (University of London)), 1974.

12. Issakhanian, E., Elkins, C.J., Lo, K.P. and Eaton, J.K. An experimental study of the flow around a formula one racing car tire, J Fluids Engineering, 132, (7).

13. Saddington, A.J., Knowles, R.D. and Knowles, K. Laser Doppler anemometry measurements in the near-wake of an isolated Formula One wheel, Experiments in fluids, 2007, 42, (5), pp 671-681.

14. Axerio-Cilies, J., Issakhanian, E., Jimenez, J. and Iaccarino, G. An aerodynamic investigation of an isolated stationary Formula 1 wheel assembly, J Fluids Engineering, 2012, 134, (2).

15. Dassanayake, P.R.K., Ramachandran, D., Salati, L., Barber, T. J. and Doig, G.C. Unsteady computational simulation of the flow structure of an isolated wheel in contact with the ground. 2012, Proc. 18th 
Australasian Fluid Mechanics Conference (AFMC), Australasian Fluid Mechanics Society (AFMS), Lauceston, Australia.

16. Zhang, X., Toet, W. and Zerihan, J. Ground effect aerodynamics of racing cars, Applied Mechanics Review, 2006, 59, (1), 33-49.

17. Kellar, W.P., Targett, G.J., Savill, A.M. and Dawes, W.N. An Investigation of FlowfieldInfluences Around the Front Wheel of a Formula 1 Car. Proc. 3rd Int Conf on the Engineering of Sport, 2000, pp 353-360, June, Sheffield, UK.

18. Barber T.J., Leonardi E. and Archer R.D. Causes for discrepancies in ground effect analyses. Aeronaut $J, 2002,106,(1066)$, pp 653-657.

19. Ranzenbach, R. and Barlow, J. Multielement Aerofoil in Ground Effect, Experimental and Computational Study, 1997, AIAA 97-2238.

20. Stapleford, W.R. and CARr, G.W. Aerodynamic Characteristics of Exposed Rotating Wheels, 1970. MIRA Technical Report 1970/2.

21. Doig, G., Barber, T.J., Leonardi, E. and Neely, A.J. The onset of compressibility effects for an inverted aerofoil in ground effect, Aeronaut $J, 2011$, 111, (1126), pp 797-806.

22. Doig, G., BARBer, T. and NeELY, A. The influence of compressibility on the aerodynamics of an inverted wing in ground effect, ASME J Fluids Eng, 2011, 133, (6), pp 1-12.

23. Keogh, J., Doig, G. and Diasinos, S. The Influence of Compressibility Effects in Correlation Issues for Aerodynamic Development of Racing Cars, 2012. Proc. 18th Australasian Fluid Mechanics Conference (AFMC), Australasian Fluid Mechanics Society (AFMS), Lauceston, Australia.

24. DoIG, G. Transonic and supersonic ground effect aerodynamics, 2014, Progress in Aerospace Sciences (in press, March 2014, http://dx.doi.org/10·1016/j.paerosci.2014.02.002).

25. KatZ, J. Aerodynamic Effects of Indy Car Components, 2002, SAE 2002-01-3311.

26. Diasinos, S. and Gatto, A. Experimental investigation into wing span and angle-of-attack effects on sub-scale race car wing/wheel interaction aerodynamics, Experiments in Fluids, 2008, 45, (3), pp 537-546.

27. Van Den Berg, M.A. and Zhang, X. The aerodynamic interaction between an inverted wing and a rotating wheel, J Fluids Engineering, 2009, 131, (10).

28. Diasinos, S. The Aerodynamic Interaction of a Rotating Wheel and a Downforce Producing Wing in Ground Effect, 2009. PhD, School of Mechanical and Manufacturing Engineering, University of New South Wales, Australia.

29. AIAA, Guide for the Verification and Validation of Computational Fluid Dynamics Simulations, AIAA, G-077-1998, 1998.

30. Roache, P.J. Quantification of uncertainty in computational fluid dynamics, Annual Review of Fluid Mechanics, 1997, 29, (1), pp 123-160.

31. Shit, T.H., Liou , W.W., Shabbir, A., YAng, Z. and Zhu, J. A new $k-\varepsilon$ eddy-viscosity model for high reynolds number turbulent flows - model development and validation, Computers \& Fluids, 1995 24, (3), pp 227-238.

32. Menter, F.R. Two-equation eddy-viscosity turbulence models for engineering applications, AIAA J, 1994, 32, (8), pp 269-289.

33. MAHON, S. and ZhANG, X. Computational analysis of pressure and wake characteristics of an aerofoil in ground effect, J Fluids Engineering, 2005, 127, (2), pp 290-298.

34. McManus, J. and Zhang, X. A computational study of the flow around an isolated wheel in contact with the ground, J Fluids Engineering, 2006, 128, (3), pp 520-530. 